\title{
A cross-sectional population-based survey of migraine and headache in 21,177 Norwegians: the Akershus sleep apnea project
}

\author{
Michael Bjørn Russell · Håvard Anton Kristiansen • \\ Jūratė Šaltytė-Benth · Kari Jorunn Kværner
}

Received: 1 July 2008/Accepted: 19 September 2008/Published online: 11 October 2008

(C) Springer-Verlag 2008

\begin{abstract}
The objective was to investigate the prevalence and interrelation of migraine and headache in the general population. Forty thousand men and women aged 20-80 years from the Norwegian general population received a mailed questionnaire with questions about migraine and headache. The questionnaire response rate was $54.5 \%$. The lifetime prevalence of migraine was $26.5 \%$ (95\% CI $25.9-27.1 \%$ ), i.e., $18.1 \%$ (95\% CI $17.3-18.9 \%$ ) in men and $34.1 \%$ (95\% CI $33.2-35.0 \%)$ in women. The prevalence of migraine decreased slightly in both men and women after an age of 45 . The 1 year prevalence of headache was $77.2 \%$, i.e., $69.6 \%$ (95\% CI $68.7-70.6 \%$ ) in men and $84.0 \%$ (95\% CI $83.3-84.7 \%$ ) in women. The
\end{abstract}

M. B. Russell ( $\square)$ · H. A. Kristiansen · K. J. Kværner

Head and Neck Research Group,

Research Centre, Akershus University Hospital,

1478 Lørenskog, Oslo, Norway

e-mail: m.b.russell@medisin.uio.no

M. B. Russell · H. A. Kristiansen · J. Šaltytè-Benth .

K. J. Kværner

Faculty Division Akershus University Hospital,

University of Oslo, 1474 Nordbyhagen, Oslo, Norway

M. B. Russell

Department of Neurology,

Akershus University Hospital,

1478 Lørenskog, Oslo, Norway

J. Šaltytè-Benth

Helse Øst Health Services, Research Centre,

Akershus University Hospital, 1478 Lørenskog, Oslo, Norway

K. J. Kværner

Department of Special Needs Eduction,

University of Oslo, Blindern, 0318 Oslo, Norway frequency of headache decreased with age, but some of the elders aged 70 or above experienced more frequent headache. The prevalence of being headache free increased from $19.1 \%$ (95\% CI $14.2-25.6 \%$ ) to $74.7 \%$ (95\% CI $70.0-78.8 \%$ ) in 20 and 80 years old men without cooccurrence of migraine, and from $5.1 \%$ (95\% CI 2.9-8.8\%) to $61.4 \%$ (95\% CI 54.9-67.6\%) in 20 and 80 years old women without co-occurrence of migraine. Co-occurrence of migraine significantly increased the frequency of headache and decreased the prevalence of being headache free.

Keywords Headache $\cdot$ Migraine $\cdot$ Epidemiology ·

Prevalence $\cdot$ Age $\cdot$ General population

\section{Introduction}

Headache is the most common type of pain experienced by man. Even though headache receives little attention probably due to the often mild symptoms, the fact is that most people rarely consult their physician for headache [1, 2]. However, the high prevalence and the considerable socioeconomic impact render that suffering from headache should not be ignored [2-4]. The most common types of headache, such as migraine and tension-type headache, cause 270 days and 820 days of work absence per year per 1,000 people [2]. "Lifting the burden", a global campaign to reduce the burden of headache worldwide concluded that headache is among the ten most disabling conditions in the World Health Organizations ranking [4].

The aim of this study is to provide epidemiological data on migraine and headache among 20-80-year- old people from the Norwegian general population. 


\section{Subjects and methods}

Sampling and representativeness

A random sample of 40,000 persons aged 20, 25, 30, 35, $40,45,50,55,60,65,70,75$ and 80 years was drawn by the National Population Register. The sample was stratified by age and gender, i.e., each age group included 2,000 persons of each gender, aged 30-60 years, while the remaining age groups included 1,000 persons of each gender. The participants were residing in Akershus, Hedmark or Oppland Counties, excluding the municipalities Asker and Bærum and municipalities north of Trysil, Åmot, Ringsaker, Vestre Slidre, Østre Slidre, Gausdal and Øyer. The sample size was reduced to 38,873 owing to decease $(n=75)$, dementia $(n=23)$, multihandicapped $(n=4)$, insufficient Norwegian skills $(n=3)$, and an error in the address list $(n=1,024)$.

The three counties have both rural and urban areas and Akershus County is situated in close proximity to Oslo. Data from Statistics Norway show that the sampling area was representative for the total Norwegian population regarding age, gender and marital status. Regarding employment; trade, hotel/restaurant and transport were overrepresented, while industry, oil and gas and financial services were underrepresented in the sampling area compared to the total Norwegian population [5].

\section{Questionnaire}

All persons received a mailed questionnaire with a standard letter containing information about the project. Apart from ensuring confidentiality and emphazising the importance of

Table 1 Questionnaire response rates by age and gender

\begin{tabular}{lll}
\hline Age & $\begin{array}{l}\text { Men } \\
\%(95 \% \text { CI })\end{array}$ & $\begin{array}{l}\text { Women } \\
\%(95 \% \text { CI })\end{array}$ \\
\hline 20 & $30.3(27.4-33.3)$ & $43.6(40.4-46.9)$ \\
25 & $30.7(27.8-33.8)$ & $46.3(43.1-49.6)$ \\
30 & $38.8(36.5-41.0)$ & $49.0(46.8-51.3)$ \\
35 & $42.5(40.2-44.7)$ & $52.7(50.4-54.9)$ \\
40 & $50.7(48.4-52.9)$ & $57.8(55.6-60.0)$ \\
45 & $51.7(49.5-54.0)$ & $57.0(54.8-59.2)$ \\
50 & $54.1(51.8-56.3)$ & $63.1(61.0-65.3)$ \\
55 & $59.0(56.8-61.2)$ & $64.3(62.2-66.4)$ \\
60 & $60.1(57.9-62.3)$ & $64.3(62.2-66.4)$ \\
65 & $70.6(67.7-73.4)$ & $67.8(64.8-70.7)$ \\
70 & $70.3(67.3-73.1)$ & $64.1(61.0-67.1)$ \\
75 & $64.5(61.4-67.4)$ & $55.7(52.5-58.8)$ \\
80 & $55.0(51.8-58.2)$ & $44.4(41.3-47.6)$ \\
Overall response rate & $51.9(51.2-52.6)$ & $57.0(56.3-57.7)$ \\
\hline
\end{tabular}

CI confidence intervals participation, it was stated that the object was to study sleepiness during daytime. The questions "Have you ever had migraine? Yes or no", and "How many days during the last year have you had headache? 0 days, 1-11 days, 1230 days, 31-84 days, $85-179$ days or $\geq 180$ days" were used to screen for migraine and headache frequency. If the questionnaire evoked no response, a second mail was issued. The replies could be either on paper or electronically. The data were collected between February and August 2006. We defined infrequent headache to be less than 12 days per year, frequent headache to be between 12 and 179 days per year, while chronic headache was 180 days or more per year [1].

\section{Statistical analyses}

All questionnaires were scanned using TeleForm v9. The statistical analyses were performed using SPSS Base System for Windows 15.0. The 95\% CI were calculated with the Vassar Collage statistics web-page [6]. $\chi^{2}$ test with $5 \%$ level of significance was used. Non-linear regression analysis was applied to explore the trends in the prevalence of different headache frequency, age and gender groups. Headache frequency can be considered as a count or ordinal variable. Firstly, the Poisson regression model was explored, when modeling the differences in headache frequency. Although the assumptions for the Poisson regression model were reasonable, the fit was rather poor in this model. Secondly, the ordinal logistic regression model was considered. However, the assumption of parallel lines was not met, and this model was rejected. Thus, the multinomial logistic regression model was estimated to

Table 2 Lifetime prevalence of migraine related to age and gender

\begin{tabular}{lll}
\hline Age & $\begin{array}{l}\text { Men } \\
\%(95 \% \mathrm{CI})\end{array}$ & $\begin{array}{l}\text { Women } \\
\%(95 \% \mathrm{CI})\end{array}$ \\
\hline 20 & $21.8(17.2-27.3)$ & $31.4(26.9-36.4)$ \\
25 & $22.6(17.8-28.3)$ & $34.5(30.0-39.4)$ \\
30 & $20.5(17.6-23.8)$ & $36.8(33.6-40-1)$ \\
35 & $21.8(19.0-25.0)$ & $37.8(34.7-40.9)$ \\
40 & $22.0(19.3-24.8)$ & $38.0(35.1-41.0)$ \\
45 & $20.6(18.1-23.4)$ & $37.4(34.5-40.4)$ \\
50 & $18.3(15.9-20.9)$ & $33.7(31.0-36.5)$ \\
55 & $18.4(16.2-20.9)$ & $35.4(32.7-38.3)$ \\
60 & $18.0(15.8-20.4)$ & $34.8(32.2-37.6)$ \\
65 & $14.1(11.6-17.1)$ & $31.0(27.4-34.8)$ \\
70 & $11.6(9.3-14.4)$ & $25.9(22.5-29.7)$ \\
75 & $13.5(10.9-16.5)$ & $24.3(20.7-28.3)$ \\
80 & $10.9(8.4-14.0)$ & $27.2(22.9-31.9)$ \\
Overall prevalence & $18.1(17.3-18.9)$ & $34.1(33.2-35.0)$ \\
\hline
\end{tabular}

CI confidence intervals 
Table 3 Headache frequency in relation to migraine, age and gender in people from the Norwegian general population

\begin{tabular}{|c|c|c|c|c|c|c|c|}
\hline \multirow{2}{*}{$\begin{array}{l}\text { Age } \\
\text { (years) }\end{array}$} & \multirow[t]{2}{*}{$N$} & \multirow{2}{*}{$\begin{array}{l}\text { No headache } \\
0 \text { days } \\
(\%)\end{array}$} & \multirow{2}{*}{$\begin{array}{l}\text { Infrequent headache } \\
1-11 \text { days } \\
(\%)\end{array}$} & \multicolumn{3}{|c|}{ Frequent headache } & \multirow{2}{*}{$\begin{array}{l}\text { Chronic headache } \\
\geq 180 \text { days } \\
(\%)\end{array}$} \\
\hline & & & & $\begin{array}{l}\text { 12-30 days } \\
(\%)\end{array}$ & $\begin{array}{l}31-84 \text { days } \\
(\%)\end{array}$ & $\begin{array}{l}85-179 \text { days } \\
(\%)\end{array}$ & \\
\hline
\end{tabular}

No migraine

Men

\begin{tabular}{|c|c|c|c|c|c|c|c|}
\hline 20 & 202 & 19.3 & 55.0 & 21.3 & 4.0 & 0.5 & 0.0 \\
\hline 25 & 197 & 15.7 & 56.9 & 17.8 & 8.6 & 1.0 & 0.0 \\
\hline 30 & 512 & 14.8 & 53.1 & 19.9 & 8.0 & 2.7 & 1.4 \\
\hline 35 & 563 & 15.5 & 56.1 & 14.9 & 8.2 & 3.0 & 2.3 \\
\hline 40 & 674 & 16.3 & 55.0 & 16.2 & 8.0 & 4.0 & 0.4 \\
\hline 45 & 700 & 20.4 & 55.6 & 12.1 & 7.7 & 3.1 & 1.0 \\
\hline 50 & 754 & 27.9 & 50.0 & 12.9 & 5.4 & 2.4 & 1.5 \\
\hline 55 & 830 & 35.3 & 45.3 & 11.9 & 4.1 & 1.6 & 1.8 \\
\hline 60 & 858 & 41.7 & 42.8 & 9.0 & 3.6 & 1.7 & 1.2 \\
\hline 65 & 517 & 50.1 & 39.7 & 6.6 & 2.1 & 0.2 & 1.4 \\
\hline 70 & 521 & 56.8 & 33.0 & 4.4 & 4.0 & 1.0 & 0.8 \\
\hline 75 & 473 & 63.8 & 25.6 & 4.7 & 4.2 & 1.1 & 0.6 \\
\hline 80 & 395 & 74.7 & 15.2 & 3.3 & 4.1 & 1.3 & 1.5 \\
\hline Total & 7,196 & 34.7 & 45.2 & 11.4 & 5.5 & 2.0 & 1.2 \\
\hline \multicolumn{8}{|c|}{ Women } \\
\hline 20 & 255 & 5.1 & 47.1 & 25.5 & 14.1 & 7.8 & 0.4 \\
\hline 25 & 262 & 7.3 & 45.4 & 28.2 & 11.5 & 5.7 & 1.9 \\
\hline 30 & 528 & 8.9 & 47.2 & 23.9 & 11.6 & 5.9 & 2.7 \\
\hline 35 & 575 & 6.8 & 45.2 & 24.2 & 13.9 & 6.8 & 3.1 \\
\hline 40 & 646 & 6.8 & 46.4 & 26.0 & 12.4 & 6.2 & 2.2 \\
\hline 45 & 644 & 10.7 & 46.7 & 24.2 & 12.4 & 3.4 & 2.5 \\
\hline 50 & 739 & 14.6 & 51.3 & 20.6 & 7.8 & 3.8 & 1.9 \\
\hline 55 & 711 & 22.6 & 48.8 & 15.5 & 8.6 & 3.1 & 1.4 \\
\hline 60 & 723 & 23.9 & 51.0 & 12.6 & 6.9 & 3.0 & 2.5 \\
\hline 65 & 399 & 34.6 & 39.3 & 11.8 & 9.0 & 3.5 & 1.8 \\
\hline 70 & 400 & 45.0 & 35.0 & 10.8 & 6.3 & 1.3 & 1.8 \\
\hline 75 & 348 & 51.4 & 33.9 & 7.8 & 5.7 & 0.6 & 0.6 \\
\hline 80 & 236 & 61.4 & 21.6 & 8.1 & 3.4 & 2.5 & 3.0 \\
\hline Total & 6,466 & 20.3 & 45.0 & 18.8 & 9.7 & 4.1 & 2.1 \\
\hline \multicolumn{8}{|c|}{ Co-occurrence of migraine } \\
\hline \multicolumn{8}{|l|}{ Men } \\
\hline 20 & 60 & 1.7 & 38.3 & 33.3 & 18.3 & 5.0 & 3.3 \\
\hline 25 & 60 & 3.3 & 36.7 & 31.7 & 18.3 & 8.3 & 1.7 \\
\hline 30 & 138 & 3.6 & 28.3 & 26.8 & 29.0 & 6.5 & 5.8 \\
\hline 35 & 162 & 3.7 & 34.6 & 35.8 & 17.3 & 4.9 & 3.7 \\
\hline 40 & 197 & 3.0 & 33.5 & 28.9 & 18.3 & 11.2 & 5.1 \\
\hline 45 & 191 & 2.1 & 35.6 & 24.1 & 23.0 & 8.9 & 6.3 \\
\hline 50 & 175 & 5.7 & 32.0 & 31.4 & 17.1 & 10.3 & 3.4 \\
\hline 55 & 193 & 13.0 & 37.8 & 24.4 & 14.0 & 7.3 & 3.6 \\
\hline 60 & 196 & 13.8 & 47.4 & 16.3 & 9.7 & 7.7 & 5.1 \\
\hline 65 & 87 & 19.5 & 50.6 & 13.8 & 11.5 & 2.3 & 2.3 \\
\hline 70 & 70 & 27.1 & 42.9 & 15.7 & 10.0 & 4.3 & 0.0 \\
\hline 75 & 74 & 33.8 & 25.7 & 14.9 & 17.6 & 4.1 & 4.1 \\
\hline 80 & 49 & 30.6 & 42.9 & 10.2 & 14.3 & 0.0 & 2.0 \\
\hline Total & 1,652 & 9.8 & 36.9 & 24.8 & 17.1 & 7.2 & 4.1 \\
\hline
\end{tabular}


Table 3 continued

\begin{tabular}{|c|c|c|c|c|c|c|c|}
\hline \multirow{2}{*}{$\begin{array}{l}\text { Age } \\
\text { (years) }\end{array}$} & \multirow[t]{2}{*}{$N$} & \multirow{2}{*}{$\begin{array}{l}\text { No headache } \\
0 \text { days } \\
(\%)\end{array}$} & \multirow{2}{*}{$\begin{array}{l}\text { Infrequent headache } \\
1-11 \text { days } \\
(\%)\end{array}$} & \multicolumn{3}{|c|}{ Frequent headache } & \multirow{2}{*}{$\begin{array}{l}\text { Chronic headache } \\
\geq 180 \text { days } \\
(\%)\end{array}$} \\
\hline & & & & $\begin{array}{l}12-30 \text { days } \\
(\%)\end{array}$ & $\begin{array}{l}31-84 \text { days } \\
(\%)\end{array}$ & $\begin{array}{l}85-179 \text { days } \\
(\%)\end{array}$ & \\
\hline \multicolumn{8}{|c|}{ Women } \\
\hline 20 & 117 & 0.9 & 24.8 & 21.4 & 30.8 & 14.5 & 7.7 \\
\hline 25 & 142 & 0.0 & 24.6 & 30.3 & 24.6 & 15.5 & 4.9 \\
\hline 30 & 323 & 1.9 & 22.9 & 31.6 & 26.0 & 10.5 & 7.1 \\
\hline 35 & 359 & 0.6 & 22.3 & 25.1 & 30.1 & 15.3 & 6.7 \\
\hline 40 & 411 & 1.0 & 19.5 & 27.3 & 29.7 & 13.1 & 9.5 \\
\hline 45 & 392 & 2.0 & 22.2 & 31.9 & 25.5 & 10.7 & 7.7 \\
\hline 50 & 383 & 3.4 & 27.9 & 25.8 & 22.5 & 13.1 & 7.3 \\
\hline 55 & 405 & 5.4 & 33.1 & 28.1 & 21.0 & 7.9 & 4.4 \\
\hline 60 & 408 & 8.3 & 39.0 & 21.6 & 16.9 & 7.4 & 6.9 \\
\hline 65 & 181 & 15.5 & 37.0 & 23.2 & 13.3 & 7.2 & 3.9 \\
\hline 70 & 146 & 26.7 & 32.2 & 18.5 & 17.8 & 2.7 & 2.1 \\
\hline 75 & 112 & 31.3 & 34.8 & 11.6 & 12.5 & 4.5 & 5.4 \\
\hline 80 & 92 & 39.1 & 21.7 & 13.0 & 16.3 & 5.4 & 4.3 \\
\hline Total & 3,471 & 6.6 & 27.6 & 25.7 & 23.2 & 10.5 & 6.5 \\
\hline
\end{tabular}

The sum of each row is $100 \%$

quantify the differences in headache frequency in relation to age, gender, age and co-occurrence of migraine.

Ethical issues

The Regional Committees for Medical Research Ethics and the Norwegian Social Science Data Services approved the project.

\section{Results}

Questionnaire response rate

The overall response rate was $54.5 \%$, and it was significantly higher among women than men $(P<0.001)$. Table 1 shows the response rate by age and gender. The response rate increased steadily from age 20 to age 65 years, and then it slowly declined in the elder age groups. Among responders, the first, second, and electronic questionnaire were replied by $64.7,27.0$ and $8.3 \%$, respectively. There were no significant differences between headache and migraine response to first, second and electronic version analyzed separately by age and gender. The electronic version was replied significantly by more men than women (men $11.6 \%$ vs. women 5.3\%; $P<0.001$ ), and the response rate increased slightly from age 20 to age 25 , then it declined gradually to 1.0 and $0.0 \%$ among 80 old men and women $(P<0.001$ in both gender). Information about headache was missing in $5.9 \%$ of men and
$4.8 \%$ of women, while information about migraine was missing in $6.6 \%$ of men and $5.2 \%$ of women. Complete information on both headache and migraine was received by $88.0 \%$ of men and $89.4 \%$ of women.

\section{Migraine}

Table 2 shows the prevalence of migraine by age and gender. Women had significantly more frequent migraine than men [34.1\% (95\% CI 33.2-35.0\%) vs. $18.1 \%$ (95\% CI $25.9-27.1 \%), P<0.001]$. The prevalence in men was unchanged from age 20 to age 40 years, and then it decreased slightly with age, while the prevalence in women increased from age 20 to age 40 years, and then decreased slightly with increasing age.

\section{Headache}

The 1 year prevalence of headache was $77.2 \%$, i.e., $69.6 \%$ (95\% CI 68.7-70.6\%) in men and $84.0 \%$ (95\% CI $83.3-$ $84.7 \%$ ) in women. Table 3 shows headache frequency in relation to migraine, age and gender. The frequency of headache decreased with age, but some of the elders aged 70 years or above experienced more frequent headache. The risk and frequency of headache was significantly higher in those with than without co-occurrence of migraine $(P<0.001$ for both the gender $)$. Table 4 presents the results of a multinomial logistic regression model showing odds ratios in relation to headache frequency, age and gender, with headache free and age 
Table 4 Headache analyzed with a multinomial logistic regression model

\begin{tabular}{|c|c|c|c|c|c|c|}
\hline \multirow[t]{2}{*}{ Age and migraine } & \multicolumn{3}{|l|}{ Men } & \multicolumn{3}{|l|}{ Women } \\
\hline & Odds ratios & $95 \% \mathrm{CI}$ & $P$ values & Odds ratios & $95 \% \mathrm{CI}$ & $P$ values \\
\hline \multicolumn{7}{|l|}{ 1-11 days } \\
\hline 20 & 0.879 & $0.583-1.324$ & 0.537 & 1.384 & $0.741-2.587$ & 0.308 \\
\hline 25 & 1.069 & $0.693-1.649$ & 0.764 & 1.036 & $0.589-1.820$ & 0.903 \\
\hline 30 & 1.031 & $0.749-1.420$ & 0.850 & 0.768 & $0.505-1.167$ & 0.216 \\
\hline 35 & 1.062 & $0.782-1.444$ & 0.700 & 1.051 & $0.675-1.635$ & 0.827 \\
\hline 40 & 1 & - & - & 1 & - & - \\
\hline 45 & 0.826 & $0.626-1.090$ & 0.176 & 0.634 & $0.430-0.934$ & 0.021 \\
\hline 50 & 0.527 & $0.405-0.684$ & $<0.001$ & 0.508 & $0.354-0.729$ & $<0.001$ \\
\hline 55 & 0.373 & $0.290-0.480$ & $<0.001$ & 0.325 & $0.230-0.459$ & $<0.001$ \\
\hline 60 & 0.312 & $0.244-0.400$ & $<0.001$ & 0.313 & $0.223-0.441$ & $<0.001$ \\
\hline 65 & 0.239 & $0.183-0.313$ & $<0.001$ & 0.169 & $0.117-0.242$ & $<0.001$ \\
\hline 70 & 0.173 & $0.132-0.227$ & $<0.001$ & 0.107 & $0.075-0.154$ & $<0.001$ \\
\hline 75 & 0.113 & $0.085-0.151$ & $<0.001$ & 0.092 & $0.064-0.133$ & $<0.001$ \\
\hline 80 & 0.070 & $0.051-0.096$ & $<0.001$ & 0.048 & $0.032-0.073$ & $<0.001$ \\
\hline Migraine & 2.877 & $2.385-3.470$ & $<0.001$ & 1.981 & $1.679-2.337$ & $<0.001$ \\
\hline No migraine & 1 & - & - & 1 & - & - \\
\hline \multicolumn{7}{|l|}{ 12-30 days } \\
\hline 20 & 1.065 & $0.664-1.708$ & 0.793 & 1.198 & $0.629-2.281$ & 0.583 \\
\hline 25 & 1.117 & $0.675-1.847$ & 0.667 & 1.090 & $0.612-1.942$ & 0.769 \\
\hline 30 & 1.241 & $0.858-1.793$ & 0.252 & 0.732 & $0.476-1.127$ & 0.157 \\
\hline 35 & 1.068 & $0.746-1.530$ & 0.719 & 0.965 & $0.612-1.521$ & 0.879 \\
\hline 40 & 1 & - & - & 1 & - & - \\
\hline 45 & 0.624 & $0.444-0.877$ & 0.007 & 0.619 & $0.415-0.923$ & 0.019 \\
\hline 50 & 0.494 & $0.358-0.680$ & $<0.001$ & 0.357 & $0.244-0.521$ & $<0.001$ \\
\hline 55 & 0.317 & $0.231-0.434$ & $<0.001$ & 0.198 & $0.137-0.286$ & $<0.001$ \\
\hline 60 & 0.190 & $0.137-0.263$ & $<0.001$ & 0.138 & $0.095-0.199$ & $<0.001$ \\
\hline 65 & 0.116 & $0.078-0.173$ & $<0.001$ & 0.089 & $0.059-0.134$ & $<0.001$ \\
\hline 70 & 0.079 & $0.051-0.121$ & $<0.001$ & 0.054 & $0.036-0.082$ & $<0.001$ \\
\hline 75 & 0.070 & $0.045-0.109$ & $<0.001$ & 0.032 & $0.020-0.051$ & $<0.001$ \\
\hline 80 & 0.042 & $0.024-0.071$ & $<0.001$ & 0.028 & $0.017-0.045$ & $<0.001$ \\
\hline Migraine & 7.666 & $6.230-9.433$ & $<0.001$ & 4.523 & $3.797-5.387$ & $<0.001$ \\
\hline No migraine & 1 & - & - & 1 & - & - \\
\hline \multicolumn{7}{|l|}{ 31-84 days } \\
\hline 20 & 0.587 & $0.314-1.096$ & 0.094 & 1.389 & $0.717-2.691$ & 0.330 \\
\hline 25 & 1.060 & $0.589-1.909$ & 0.846 & 0.854 & $0.464-1.569$ & 0.610 \\
\hline 30 & 1.348 & $0.884-2.058$ & 0.166 & 0.643 & $0.409-1.012$ & 0.056 \\
\hline 35 & 1.027 & $0.674-1.564$ & 0.901 & 1.103 & $0.690-1.762$ & 0.682 \\
\hline 40 & 1 & - & - & 1 & - & - \\
\hline 45 & 0.861 & $0.586-1.266$ & 0.447 & 0.546 & $0.359-0.832$ & 0.005 \\
\hline 50 & 0.428 & $0.289-0.635$ & $<0.001$ & 0.284 & $0.190-0.426$ & $<0.001$ \\
\hline 55 & 0.243 & $0.163-0.362$ & $<0.001$ & 0.174 & $0.118-0.257$ & $<0.001$ \\
\hline 60 & 0.158 & $0.105-0.239$ & $<0.001$ & 0.122 & $0.082-0.182$ & $<0.001$ \\
\hline 65 & 0.097 & $0.057-0.166$ & $<0.001$ & 0.082 & $0.053-0.128$ & $<0.001$ \\
\hline 70 & 0.121 & $0.074-0.197$ & $<0.001$ & 0.054 & $0.035-0.085$ & $<0.001$ \\
\hline 75 & 0.129 & $0.081-0.206$ & $<0.001$ & 0.037 & $0.023-0.061$ & $<0.001$ \\
\hline 80 & 0.099 & $0.059-0.166$ & $<0.001$ & 0.027 & $0.016-0.048$ & $<0.001$ \\
\hline Migraine & 11.168 & $8.896-14.021$ & $<0.001$ & 7.978 & $6.633-9.596$ & $<0.001$ \\
\hline No migraine & 1 & - & - & 1 & - & - \\
\hline
\end{tabular}


Table 4 continued

\begin{tabular}{|c|c|c|c|c|c|c|}
\hline \multirow[t]{2}{*}{ Age and migraine } & \multicolumn{3}{|l|}{ Men } & \multicolumn{3}{|l|}{ Women } \\
\hline & Odds ratios & $95 \% \mathrm{CI}$ & $P$ values & Odds ratios & $95 \% \mathrm{CI}$ & $P$ values \\
\hline \multicolumn{7}{|l|}{ 85-179 days } \\
\hline 20 & 0.226 & $0.076-0.672$ & 0.007 & 1.542 & $0.755-3.151$ & 0.235 \\
\hline 25 & 0.486 & $0.199-1.185$ & 0.112 & 1.046 & $0.540-2.028$ & 0.893 \\
\hline 30 & 0.706 & $0.396-1.260$ & 0.239 & 0.620 & $0.372-1.031$ & 0.066 \\
\hline 35 & 0.637 & $0.363-1.118$ & 0.116 & 1.186 & $0.711-1.979$ & 0.514 \\
\hline 40 & 1 & - & - & 1 & - & - \\
\hline 45 & 0.630 & $0.384-1.033$ & 0.067 & 0.417 & $0.256-0.679$ & $<0.001$ \\
\hline 50 & 0.400 & $0.244-0.655$ & $<0.001$ & 0.331 & $0.210-0.522$ & $<0.001$ \\
\hline 55 & 0.197 & $0.117-0.333$ & $<0.001$ & 0.138 & $0.086-0.220$ & $<0.001$ \\
\hline 60 & 0.174 & $0.104-0.289$ & $<0.001$ & 0.114 & $0.072-0.183$ & $<0.001$ \\
\hline 65 & 0.026 & $0.008-0.084$ & $<0.001$ & 0.079 & $0.046-0.137$ & $<0.001$ \\
\hline 70 & 0.064 & $0.029-0.140$ & $<0.001$ & 0.021 & $0.010-0.044$ & $<0.001$ \\
\hline 75 & 0.058 & $0.026-0.126$ & $<0.001$ & 0.017 & $0.007-0.038$ & $<0.001$ \\
\hline 80 & 0.040 & $0.015-0.103$ & $<0.001$ & 0.028 & $0.014-0.057$ & $<0.001$ \\
\hline Migraine & 12.664 & $9.413-17.037$ & $<0.001$ & 8.552 & $6.856-10.667$ & $<0.001$ \\
\hline No migraine & 1 & - & - & 1 & - & - \\
\hline \multicolumn{7}{|l|}{$\geq 180$ days } \\
\hline 20 & 0.426 & $0.092-1.984$ & 0.277 & 0.751 & $0.303-1.862$ & 0.536 \\
\hline 25 & 0.262 & $0.033-2.084$ & 0.205 & 0.605 & $0.264-1.388$ & 0.236 \\
\hline 30 & 1.733 & $0.778-3.861$ & 0.178 & 0.625 & $0.349-1.117$ & 0.113 \\
\hline 35 & 1.826 & $0.852-3.912$ & 0.122 & 0.941 & $0.523-1.695$ & 0.839 \\
\hline 40 & 1 & - & - & 1 & - & - \\
\hline 45 & 1.156 & $0.545-2.452$ & 0.705 & 0.531 & $0.309-0.913$ & 0.022 \\
\hline 50 & 0.711 & $0.332-1.523$ & 0.381 & 0.316 & $0.186-0.539$ & $<0.001$ \\
\hline 55 & 0.606 & $0.294-1.250$ & 0.175 & 0.126 & $0.071-0.221$ & $<0.001$ \\
\hline 60 & 0.437 & $0.210-0.912$ & 0.027 & 0.177 & $0.106-0.296$ & $<0.001$ \\
\hline 65 & 0.289 & 0.119-0.699 & 0.006 & 0.073 & $0.037-0.143$ & $<0.001$ \\
\hline 70 & 0.120 & $0.038-0.378$ & $<0.001$ & 0.041 & 0.019-0.086 & $<0.001$ \\
\hline 75 & 0.163 & $0.060-0.442$ & $<0.001$ & 0.034 & $0.015-0.076$ & $<0.001$ \\
\hline 80 & 0.209 & $0.080-0.541$ & 0.001 & 0.049 & $0.024-0.103$ & $<0.001$ \\
\hline Migraine & 12.169 & $8.498-17.425$ & $<0.001$ & 10.358 & $7.967-13.467$ & $<0.001$ \\
\hline No migraine & 1 & - & - & 1 & - & - \\
\hline
\end{tabular}

40 years old as reference categories. Intercept was included in the model, but is not show in the table. The Pearson $\chi^{2}$ values were insignificant for both the models indicating that the estimated multinomial regression models fit data reasonably well. Table 5 shows age trends in different subgroups assessed using the non-linear regression model for each gender separately. This fit was rather good, with moderate or high values of $R^{2}$ and normally distributed residuals. In some subgroups the linear regression gave best fit, however,graphical analysis of the data showed that most subgroups displayed a curvilinear relationship (second or third order) between headache frequency and age (Fig. 1).
Headache free

The prevalence of being headache free increased from $19.1 \%$ (95\% CI $14.2-25.6 \%$ ) to $74.7 \%$ (95\% CI 70.0 $78.8 \%$ ) in 20 and 80 -year-old men without co-occurrence of migraine, and from $5.1 \%$ (95\% CI 2.9-8.8\%) to $61.4 \%$ (95\% CI 54.9-67.6\%) in 20 and 80-year-old women without co-occurrence of migraine. The prevalence of being headache free increased from $1.7 \%$ (95\% CI $0.1-$ $10.1 \%$ ) to $30.6 \%$ (95\% CI $18.7-45.6 \%$ ) in 20 and 80 -yearold men with co-occurrence of migraine, and from $8.5 \%$ (95\% CI $0.4-53.6 \%$ ) to $39.1 \%$ (95\% CI $29.3-49.9 \%$ ) in 20 and 80-year-old women with co-occurrence of migraine. 
Table 5 The age trends in different subgroups were assessed using the non-linear regression model for each gender separately

\begin{tabular}{|c|c|c|c|c|c|c|}
\hline \multirow{2}{*}{$\begin{array}{l}\text { Dependent } \\
\text { variable }\end{array}$} & \multicolumn{4}{|c|}{ Coefficients of regression model } & \multirow[t]{2}{*}{$P$ values } & \multirow[t]{2}{*}{$R^{2}$} \\
\hline & $b_{0}$ & $b_{1}$ & $b_{2}$ & $b_{3}$ & & \\
\hline \multicolumn{7}{|l|}{ No migraine } \\
\hline \multicolumn{7}{|l|}{ Men } \\
\hline 0 days & 30.43 & -1.12 & 0.02 & - & $<0.001$ & 0.988 \\
\hline $1-11$ days & 39.34 & 1.07 & -0.02 & - & $<0.001$ & 0.985 \\
\hline 12-30 days & 27.03 & -0.30 & - & - & $<0.001$ & 0.957 \\
\hline 31-84 days & -19.05 & 1.94 & -0.04 & 0.0003 & $<0.001$ & 0.869 \\
\hline 85-179 days & -15.38 & 1.20 & -0.02 & 0.0001 & $<0.001$ & 0.855 \\
\hline$\geq 180$ days & -5.54 & 0.40 & -0.01 & 0.00004 & 0.197 & 0.390 \\
\hline \multicolumn{7}{|l|}{ Women } \\
\hline 0 days & 23.04 & -1.21 & 0.02 & - & $<0.001$ & 0.991 \\
\hline $1-11$ days & 22.88 & 1.32 & -0.016 & - & $<0.001$ & 0.835 \\
\hline 12-30 days & 29.49 & -0.03 & -0.003 & - & $<0.001$ & 0.924 \\
\hline 31-84 days & 17.41 & -0.16 & - & - & $<0.001$ & 0.822 \\
\hline 85-179 days & 9.20 & -0.10 & - & - & $<0.001$ & 0.821 \\
\hline$\geq 180$ days & -9.89 & 0.83 & -0.02 & 0.0001 & 0.056 & 0.550 \\
\hline \multicolumn{7}{|c|}{ Co-occurrence of migraine } \\
\hline \multicolumn{7}{|c|}{ Men } \\
\hline 0 days & 11.55 & -0.62 & 0.01 & - & $<0.001$ & 0.949 \\
\hline $1-11$ days & 89.72 & -4.18 & 0.09 & -0.001 & 0.306 & 0.317 \\
\hline 12-30 days & 43.30 & -0.39 & - & - & $<0.001$ & 0.804 \\
\hline 31-84 days & -19.61 & 3.15 & -0.07 & 0.0005 & 0.036 & 0.596 \\
\hline 85-179 days & -2.99 & 0.54 & -0.006 & - & 0.005 & 0.660 \\
\hline$\geq 180$ days & -0.004 & 0.20 & -0.002 & - & 0.199 & 0.276 \\
\hline \multicolumn{7}{|l|}{ Women } \\
\hline 0 days & 21.80 & -1.33 & 0.02 & - & $<0.001$ & 0.980 \\
\hline 1-11 days & 94.78 & -5.62 & 0.13 & -0.001 & $<0.001$ & 0.832 \\
\hline 12-30 days & 11.94 & 0.87 & -0.01 & - & $<0.001$ & 0.809 \\
\hline 31-84 days & 36.41 & -0.29 & - & - & $<0.001$ & 0.778 \\
\hline 85-179 days & 19.68 & -0.20 & - & - & $<0.001$ & 0.796 \\
\hline$\geq 180$ days & 9.00 & -0.06 & - & - & 0.037 & 0.338 \\
\hline
\end{tabular}

\section{Discussion}

Methodological considerations

The study included 21,177 persons at age 20-80 years, providing sufficient data for age and gender-specific prevalence of headache and migraine. Participation was restricted to those responding to the migraine and headache questions. The response rate was fair and there were few missing values. We were not able to investigate whether responder and non-responders had a similar migraine and headache pattern, since we were not allowed to contact non-responders. However, similar replies to the first and second issued questionnaire as well as electronic responses suggest that responders and non-responders are not different. A previous Danish epidemiological survey found no significant difference in the frequency of migraine among responders and non-responders [7]. The single question about lifetime occurrence of migraine has been evaluated against a clinical interview by two physicians in another Norwegian survey conducted in eastern Akershus County. The observed agreement rate was 0.81 and Kappa was 0.62 , a good strength of agreement (previous unpublished data). This is in line with validation of two Danish epidemiological surveys where the observed agreement rate was 0.92 and 0.94 , and Kappa was 0.77 and 0.81 , respectively $[7,8]$. A single questionnaire question about tension-type headache has previously been evaluated against a clinical interview by a physician in a Danish population-based survey [8]. The observed agreement rate was 0.91 and the chance corrected agreement rate Kappa was 0.74 , i.e., a good strength of agreement. The frequency of tension-type headache among 475 persons, aged 25-64-year- old, from the Danish general population, i.e., none, infrequent, frequent and chronic tension-type headache was also evaluated against a clinical interview by a physician on the same day in those with self-reported tension-type headache. The observed agreement rate was 0.87 and the chance corrected agreement rate Kappa was 0.77 , again a good strength of agreement [9]. Our question regarding headache is likely to be more or at least equally precise as a single question about tension-type headache, since everybody knows what headache is, while some may not know what tension-type headache is. Thus, our simple questionnaire on migraine and headache is considered to be valid, even though our epidemiological survey included persons aged 20-80 years. It would be quite surprising, if 20 years old and 70-80 years old were significantly different from 25 to 65 years old regarding our simple questions about migraine and headache. In contrast, questionnaires based on a series of questions are not valid [8]. Due to the high number of participants in our survey, it was not feasible to apply the gold standard, i.e., a clinical interview by a physician/ neurologist with expertise in headache diagnostics. The prevalence was based on headache within the last year. This was chosen in order to reduce recall bias. As headache is usually not a disabling or life threatening disorder, recall bias is likely to occur if one asks about headache years ago. Actually, the use of the 1-year prevalence is likely to secure more precise data on headache. Thus, the methodological precautions should secure that the data are both representative and generalizable.

\section{Migraine}

One of our main findings was the $26.5 \%$ lifetime prevalence of migraine with a male-female sex ratio of approximately 1:2. Our lifetime prevalence is quite similar to that found in other epidemiological surveys of 
Fig. 1 Headache frequency versus age in men (first row) and women (second row)
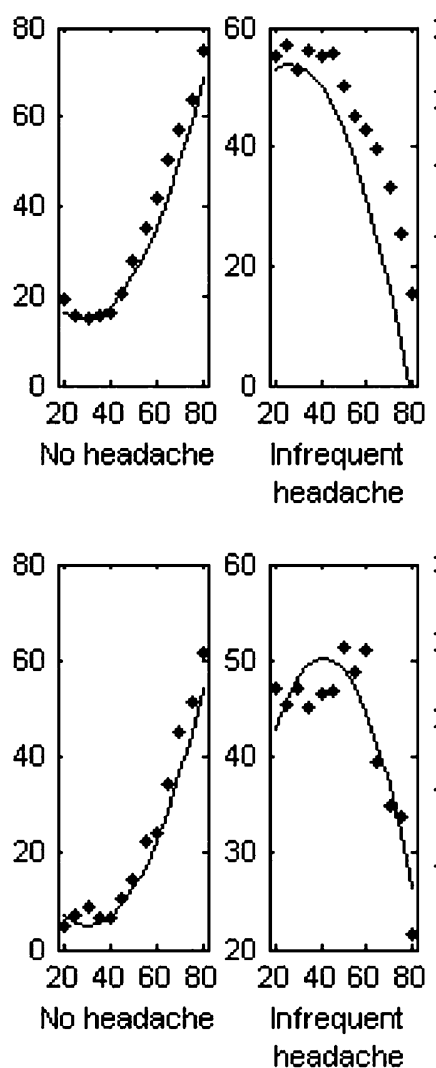
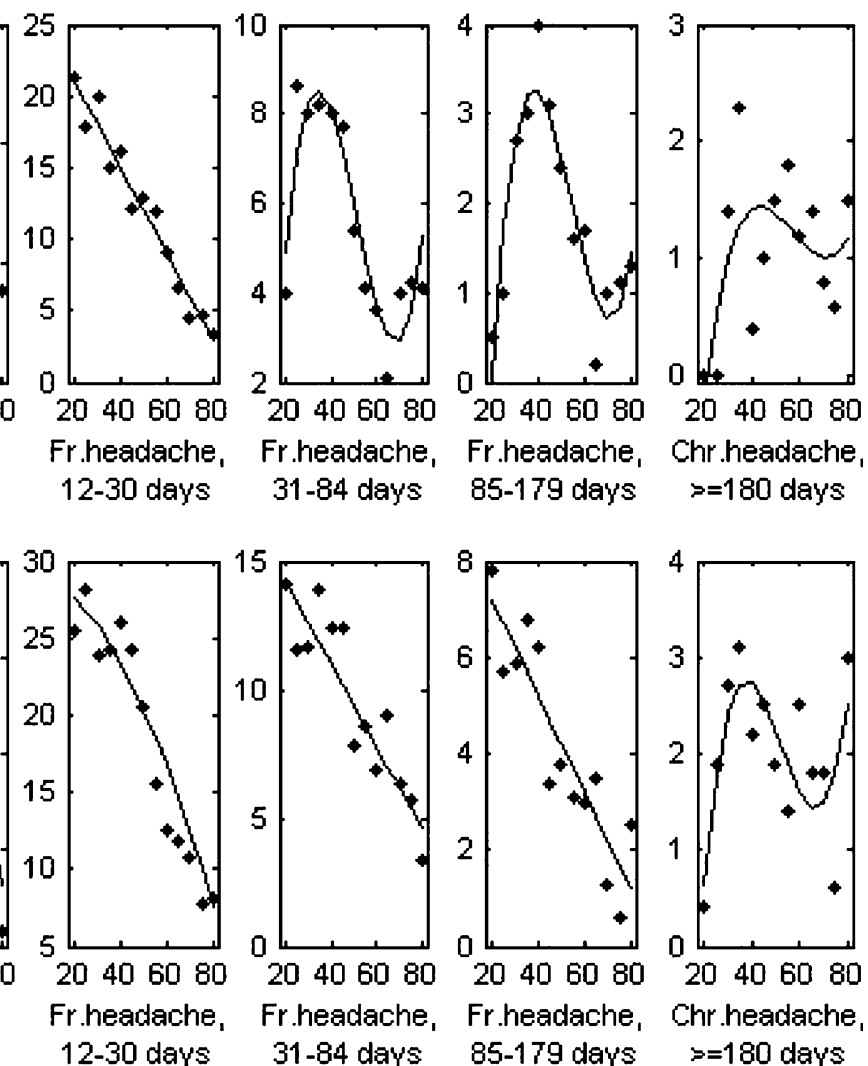
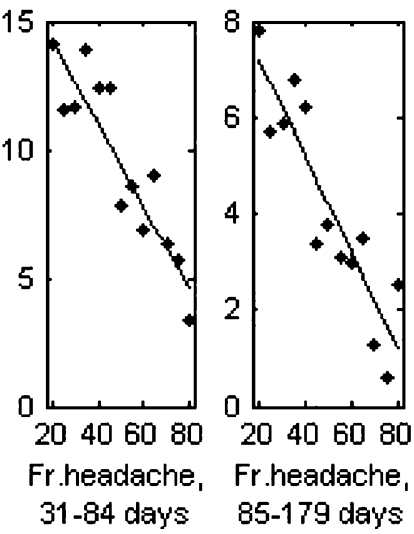

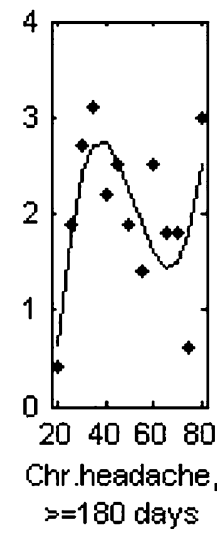

Caucasians conducted in Germany, the Netherlands and Sweden, while it is higher than that found in Canada, Croatia, Denmark and Italy [7, 10-15]. The different methodologies of these epidemiological surveys are likely to explain a part of the variation in prevalence. The prevalence of migraine is much lower in Africans, Asians and South Americans [4]. Although we do not have data on the frequency of migraine attacks, the high prevalence indicate that many people experience migraine at least a few times in their life, supporting the high socioeconomic impact of migraine [2-4]. The statistically significantly decreased prevalence of migraine in elder men can be a true phenomenon or it might be caused by recall bias. Women also had a tendency of decreased prevalence by age, but this was not statistically significant. Since, the epidemiological survey was population-based, the prevalence finding cannot be explained by physician consultation rate differences.

\section{Headache}

Another main finding was the $77.2 \%$ 1-year prevalence of headache with a male-female sex ratio of approximately 1:1.2. This corresponds well with the prevalence found in neighboring countries Denmark, Finland and Sweden [1618]. However, another Norwegian epidemiological survey found a much lower prevalence of headache [19]. The latter survey was based on the question have you suffered from headache during the last 12 months? [20]. This phrasing is likely to cause underestimation of the "real" prevalence of headache, as people with a mild headache might not reply yes to question about suffering from headache. An interesting finding was the slightly increased risk of frequent and chronic tension-type in some of the elders. Figure 1 gives a graphical presentation of this aspect. This increase is likely to be secondary to other causes like stroke, tumors, etc., which become more common with age. An increase in headache frequency is a warning sign and should be dealt seriously with proper investigations. The data clearly demonstrated that cooccurrence of migraine increased the risk of headache. This has also been found in other epidemiological surveys [9, $21,22]$. Whether frequent headaches can cause attacks that mimics migraine or whether abortive attacks of migraine can be experienced as headache is uncertain. This is currently being debated among headache researchers, but it is not the scope of our paper. However, even thought the etiology is unsolved, the features of migraine and nonmigraine headache are distinct and non-overlapping, as defined by the explicit diagnostic criteria of the International Classification of Headache Disorders [1]. The prevalence of being headache free increased significantly with age, and this is more so in men than in women, and in those without co-occurrence of migraine than those with the co-occurrence of migraine. So, even thought a person 
has frequent episodes of headache and migraine there is hope at the end of the tunnel.

Acknowledgments This study was supported by a grant from East Norway Regional Health Authority. The Faculty Division Akershus University Hospital kindly provided technical support (Anita Fjellum and Gunn Seim Ekeland). Thanks to Akershus University Hospital for providing research facilities.

\section{Conflict of interest None.}

\section{References}

1. Headache Classification Subcommittee of the International Headache Society (2004) The international classification of headache disorders. Cephalalgia 24:1-160

2. Rasmussen BK, Jensen R, Olesen J (1992) Impact of headache on sickness absence and utilisation of medical services. A Danish population study. J Epidemiol Community Health 46:443-446

3. Stewart WF, Celentano DD, Linet MS (1989) Disability, physician consultation, and use of prescription medications in a population-based study of headache. Biomed Pharmacother 43:711-718

4. Stovner LJ, Hagen K, Jensen R, Katsarava Z, Lipton R, Scher A, Steiner T, Zwart JA (2007) The global burden of headache: a documentation of headache prevalence and disability worldwide. Cephalalgia 27:193-210

5. URL: http://statbank.ssb.no/statistikkbanken

6. URL: http://faculty.vassar.edu/lowry/VassarStats.html

7. Russell MB, Rasmussen BK, Thorvaldsen P, Olesen J (1995) Prevalence and sex-ratio of the subtypes of migraine. Int J Epidemiol 24:612-618

8. Rasmussen BK, Jensen R, Olesen J (1991) Questionnaire versus clinical interview in the diagnosis of headache. Headache 31:290-295

9. Russell MB, Levi N, Saltyte-Benth J, Fenger K (2006) Tensiontype headache in adolescents and adults: a population based study of 33,764 twins. Eur J Epidemiol 21:153-160
10. Göbel H, Petersen-Braun M, Soyka D (1994) The epidemiology of headache in Germany: a nationwide survey of a representative sample on the basis of the headache classification of the International Headache Society. Cephalalgia 14:97-106

11. O'Brien B, Goeree R, Streiner D (1994) Prevalence of migraine headache in Canada: a population-based survey. Int J Epidemiol 23:1020-1026

12. Launer LJ, Terwindt GM, Ferrari MD (1999) The prevalence and characteristics of migraine in a population-based cohort: the GEM study. Neurology 53:537-542

13. Mattsson P, Svärdsudd K, Lundberg PO, Westerberg CE (2000) The prevalence of migraine in women aged 40-74 years: a population-based study. Cephalalgia 20:893-899

14. Zivadinov R, Willheim K, Jurjevic A, Sepic-Grahovac D, Bucuk M, Zorzon M (2001) Prevalence of migraine in Croatia: a population-based survey. Headache 41:805-812

15. Cologno D, Manzoni GC, Drion Medical Association (2003) An epidemiological study of migraine with aura in the San Severo general population: a pilot research project of cooperation between neurologists and general practitioners. Eur Neurol 50:195-199

16. Rasmussen BK, Jensen R, Schroll M, Olesen J (1991) Epidemiology of headache in a general population: a prevalence study. J Clin Epidemiol 44:1147-1157

17. Nikiforow R (1981) Headaache in a random sample of 200 persons: a clinical study of a population in northern Finland. Cehalalgia 1:99-107

18. Dahlöf C, Linde M (2001) One-year prevalence of migraine in Sweden: a population-based study in adults. Cephalalgia 21:664671

19. Hagen K, Zwart J-A, Vatten L, Stovner LJ, Bovim G (2000) Prevalence of migraine and non-migraineous headache-headHunt, a large population-based study. Cephalalgia 20:900-906

20. Hagen K, Zwart JA, Vatten L, Stovner LJ, Bovim G (2000) HeadHUNT: validity and reliability of a headache questionnaire in a large population-based study in Norway. Cephalalgia 20:244-251

21. Ulrich V, Russell MB, Jensen R, Olesen J (1996) A comparison of tension-type headache in migraineurs and in non-migraineurs: a population-based study. Pain 67:501-506

22. Russell MB (2005) Tension-type headache in 40-year-olds: a Danish population-based sample of 4000. J Headache Pain 6:441-447 